\title{
Reconnaissance of Ground-Water Quality in the Manatí Quadrangle, Puerto Rico, August-November 1992
}

By Carlos E. Conde-Costas and Gilberto A. Rodríguez-Rodríguez

U.S. GEOLOGICAL SURVEY

Open-File Report 96-628

Prepared in cooperation with the

PUERTO RICO ENVIRONMENTAL QUALITY BOARD

PUERTO RICO AQUEDUCT AND SEWER AUTHORITY

PUERTO RICO DEPARTMENT OF NATURAL AND ENVIRONMENTAL RESOURCES 


\section{U.S. DEPARTMENT OF THE INTERIOR BRUCE BABBITT, Secretary \\ U.S. GEOLOGICAL SURVEY \\ Gordon P. Eaton, Director}

For additional information write to:

Copies of this report can be purchased from:

District Chief

U.S. Geological Survey

GSA Center, Suite 400-15

651 Federal Drive

Guaynabo, Puerto Rico 00965

U.S. Geological Survey

Branch of Information Services

Box 25286

Denver, CO 80225-0286 


\section{CONTENTS}

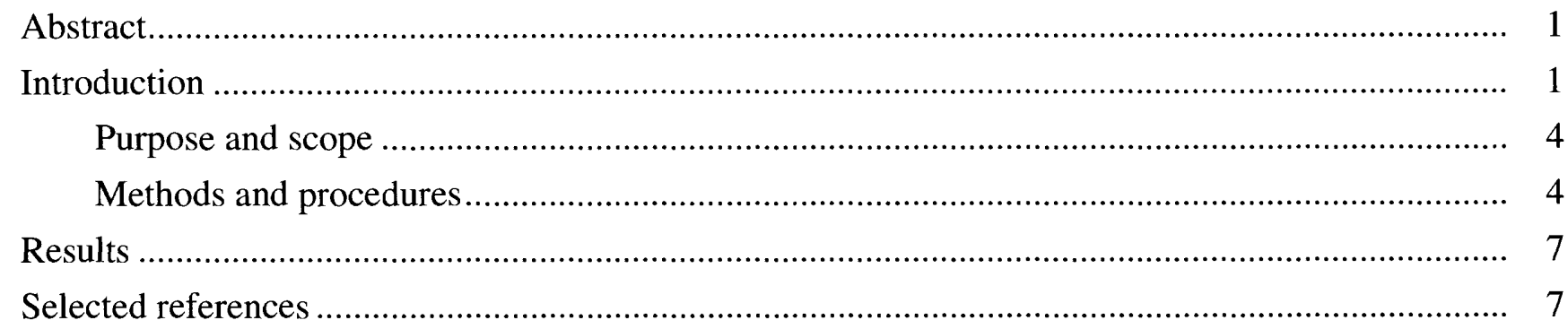

\section{FIGURES}

1. Generalized geologic map of the north coast limestone region of Puerto Rico .......................... 2

2. Generalized hydrogeologic cross section of the Manatí quadrangle, Puerto Rico........................ 3

3. Map showing location of sampling sites in the Manatí quadrangle, Puerto Rico......................... 6

\section{TABLES}

1. Description of selected surface- and ground-water sampling sites in the Manatí quadrangle, Puerto Rico, and parameters selected for laboratory analyses .....

2. Major ions, nutrients, total organic carbon, and physical properties at selected surface- and ground-water sampling sites in the Manatí quadrangle,

Puerto Rico

3. Concentrations of dissolved trace metals at selected ground-water sampling sites in the Manatí quadrangle, Puerto Rico...

4. Organic compounds analyzed in water samples collected at selected sampling sites in the Manatí quadrangle, Puerto Rico.

5. Pesticides and organic compounds detected in water from selected wells in the Manatí quadrangle, Puerto Rico

6. Stable isotope analyses at selected ground-water sampling sites in the Manatí quadrangle, Puerto Rico 
CONVERSION FACTORS, ABBREVIATED WATER-QUALITY UNITS, AND ACRONYMS

\begin{tabular}{rll}
\hline Multiply & \multicolumn{1}{c}{ By } & To obtain \\
\hline foot & 0.3048 & meter \\
mile & 1.609 & kilometer \\
square miles & 2.59 & square kilometer \\
\hline
\end{tabular}

Temperatures can be converted from degrees Celsius $\left({ }^{\circ} \mathrm{C}\right)$ to degrees Fahrenheit $\left({ }^{\circ} \mathrm{F}\right)$ by the formula:

$$
{ }^{\circ} \mathrm{F}=1.8 \times{ }^{\circ} \mathrm{C}+32
$$

Abbreviated water-quality units used in report:

$\mathrm{mg} / \mathrm{L} \quad$ milligram per liter

$\mu \mathrm{g} / \mathrm{L} \quad$ micrograms per liter

$\mu \mathrm{S} / \mathrm{cm} \quad$ microsiemens per centimeter at 25 degrees Celsius

\section{Acronyms used in report:}

MRL Minimum reporting level

USGS U.S. Geological Survey 


\title{
Reconnaissance of Ground-Water Quality in the Manatí Quadrangle, Puerto Rico, August-November 1992
}

\author{
By Carlos E. Conde-Costas and Gilberto A. Rodríguez-Rodríguez
}

\section{ABSTRACT}

A synoptic water-quality survey was conducted in the Manatí quadrangle area of Puerto Rico between August 25 and November 12, 1992. Water samples were collected at 31 sites, consisting of 29 wells, one spring, and one creek. Water-quality properties determined for these samples included: field determinations for temperature, specific conductance, $\mathrm{pH}$, and alkalinity, and laboratory analyses for dissolved constituents (cations, anions, and silica), nutrients (nitrite, nitrate plus nitrite, ammonia, phosphorous, and orthophosphorus), total organic carbon, trace metals; organochlorine insecticides and related compounds, organophosphorus insecticides, volatile organic compounds, semivolatile compounds (base, neutral aand acid methylene chloride extractable), and selected stable isotopes (oxygen-18, deuterium, nitrogen-15, and sulfur-34).

\section{INTRODUCTION}

The Manatí quadrangle area lies within the north coast limestone region (Monroe, 1980), which includes about 700 square miles of northern Puerto Rico and extends eastward from Rincón in the western part of the island to Loíza, a distance of about 85 miles (fig. 1). Three hydrogeologic units are present in the Manatí quadrangle: an upper aquifer contained in the Aymamón and Aguada Limestones of early Miocene age (fig. 2); a middle confining unit consisting of the upper member of the Cibao Formation of early Miocene age; and a lower aquifer in the Lares Limestone of Oligocene age (Rodríguez-Martínez, 1995).

The upper aquifer is the principal source of water supply in the study area. Ground water in the upper aquifer is mostly unconfined and exists as a lense of freshwater floating above a wedge of saline water and thinning toward the coast. The freshwater part of the aquifer is thickest within the Aguada Limestone where it could be as much as 300 feet thick. Within the Aymamón Limestone the thickness of the freshwater column ranges from less than 200 feet to zero at the coast. Ground-water withdrawals in the study area are 

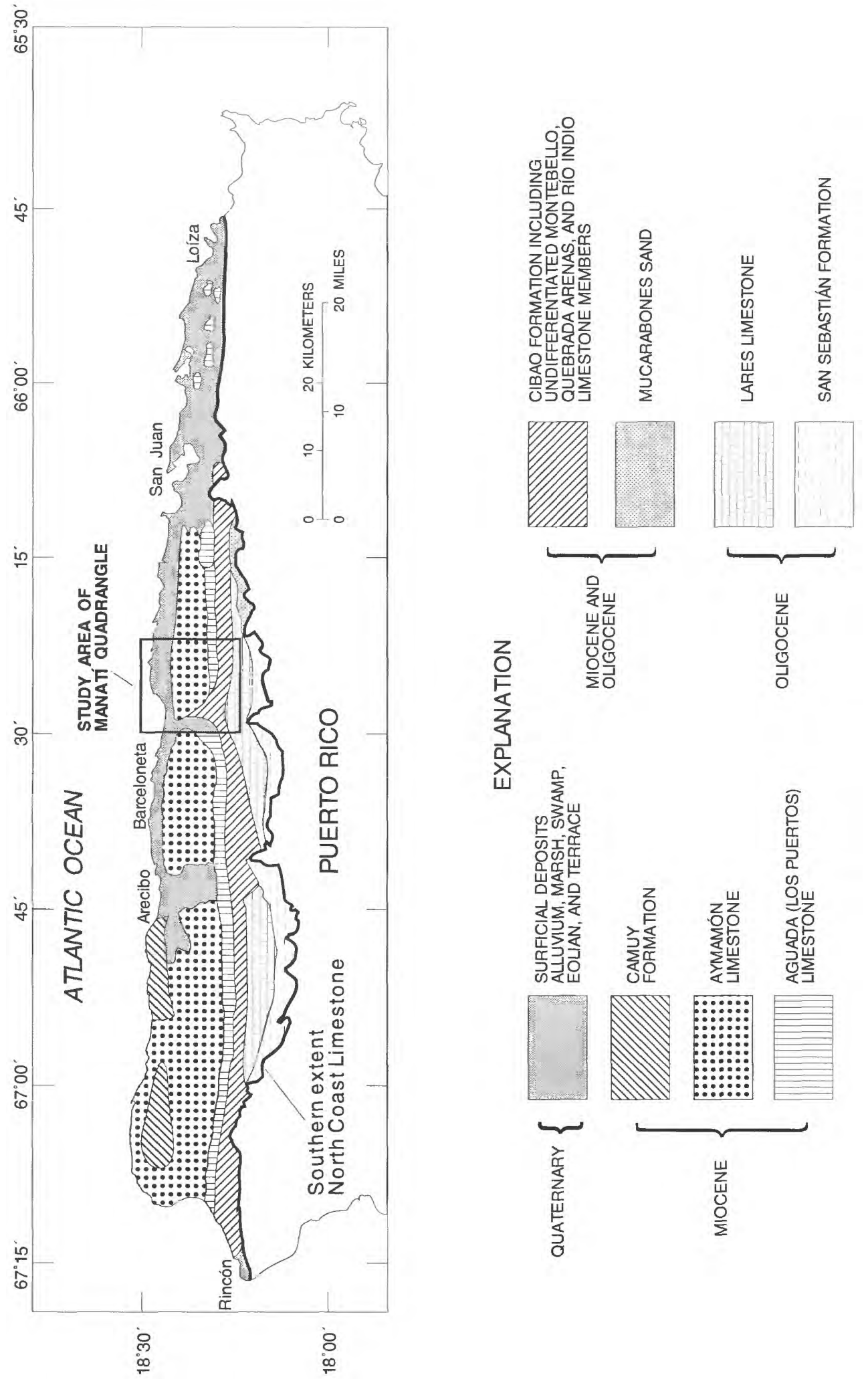

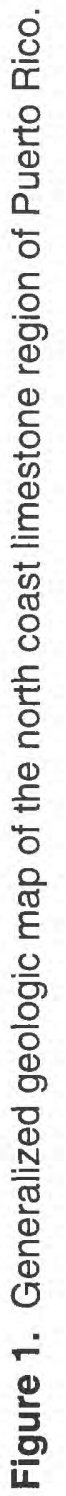



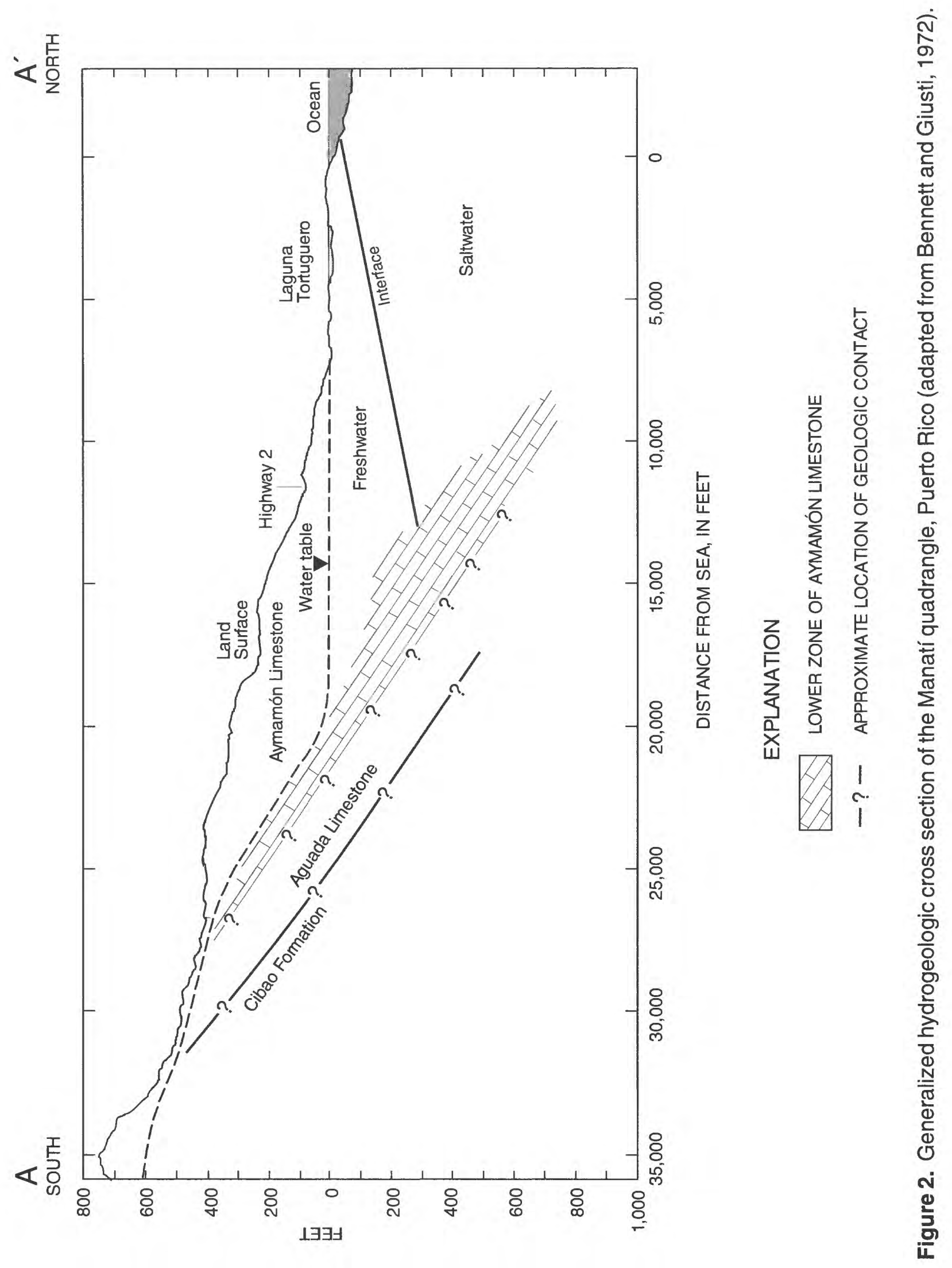
mostly limited to areas north of latitude $18^{\circ} 25^{\prime} 00^{\prime \prime} \mathrm{N}$, where the Aymamón Limestone and Aguada Limestone become saturated and constitute the upper aquifer. South of this latitude only a few shallow wells exist and these are probably screened in the Quebrada Arenas Limestone member of the Cibao Formation.

The Manatí area has been subjected to extensive agricultural, industrial, and urban development. In order to provide baseline data related to water-quality conditions in the area, the U.S. Geological Survey, in cooperation with the Puerto Rico Environmental Quality Board, the Puerto Rico Aqueduct and Sewer Authority, the Puerto Rico Department of Natural and Environmental Resources and other agencies of the Commonwealth of Puerto Rico, conducted a synoptic ground-water quality survey from August 25 to November 12, 1992.

\section{Purpose and Scope}

The purpose of the synoptic water-quality survey was to obtain data on the quality of ground water within the Manatí quadrangle, Puerto Rico. The water-quality survey covers primarily an area of approximately 20 square miles of the upper aquifer (area north of latitude $18^{\circ} 25^{\prime} \mathrm{N}$ ). However, the study area includes areas where wells tap the Quebrada Arenas Limestone, the Lares Limestone or the alluvial valley at inland parts of the quadrangle. The results from this survey are expected to be of use to water resources managers and planners, and to hydrologists for future reference and studies.

Water samples collected at 31 sites (29 wells, one spring, and one creek) were analyzed for temperature, specific conductance, $\mathrm{pH}$, alkalinity (as calcium carbonate, $\mathrm{CaCO}_{3}$ ), dissolved constituents (cations, anions, and silica), nutrients (nitrite, nitrate plus nitrite, ammonia, phosphorous and ortho-phosphorus), total organic carbon, trace metals, organochlorine insecticides and related compounds, organophosphorus insecticides, volatile organic chemicals, semivolatile compounds (base, neutral and acid methylene chloride extractable) and selected stable isotopes (oxygen-18, deuterium, nitrogen-15 and sulfur-34). Sampling sites, well characteristics and water-quality properties and constituents analyzed in the laboratory are listed in table 1.

\section{Methods and Procedures}

Water samples were collected at 31 sites, including 19 active public-water supply wells, six intermittently pumping wells (one industrial, three agricultural, and two livestock use wells), four unused wells (one flowing artesian well and three wells equipped with turbine pumps), one spring, and one small losing stream that recharges the aquifer south of latitude $18^{\circ} 25^{\prime} 00^{\prime \prime} \mathrm{N}$ (fig. 3). Water samples collected from wells were obtained at or near the well-head and prior to chlorination. Intermittently pumped wells and unused wells were sampled within a period which did not exceed 30 minutes after the pump was turned-on. Pumping time constraints imposed either by the well owner or by on-site discharge facilities limited the sampling period.

Detailed information on the procedures and methods used for on-site measurements, collecting, treating, shipping samples, and laboratory analyses are given in the following U.S. Geological Survey publications "Techniques of Water-Resources Investigations of the U.S. Geological Survey" Book 1, Chap. D2 and; Book 5, Chap. A1 and A3. Field measurements for 
Table 1. Description of selected surface- and ground-water sampling sites in the Manatí quadrangle, Puerto Rico, and parameters selected for laboratory analyses

[--, no data available; U, unused; UA, unused artesian; PS, public supply; SW, surface water; DO, domestic: AG, agricultural: LV, livestock; IN, industrial; SP, spring; A/C, anions and cations; NUT, nutrients; TM, trace metals; PST, organochlorine and organophosphorus insecticides; VOC, volatile organic compounds; SVOC, semi-volatile organic compounds: ISO. stable isotopes: Y, sample was collected: N, no sample was collected]

\begin{tabular}{|c|c|c|c|c|c|c|c|c|c|c|c|c|c|}
\hline \multirow{2}{*}{$\begin{array}{c}\text { Map } \\
\text { number }\end{array}$} & \multirow{2}{*}{$\begin{array}{l}\text { Latitude/ } \\
\text { Iongitude }\end{array}$} & \multirow{2}{*}{ Municipality } & \multirow{2}{*}{$\begin{array}{l}\text { Well name } \\
\text { or site name }\end{array}$} & \multirow{2}{*}{ Type } & \multirow{2}{*}{$\begin{array}{l}\text { Depth } \\
\text { of well } \\
\text { (feet) }\end{array}$} & \multirow{2}{*}{$\begin{array}{l}\text { Depth } \\
\text { to } \\
\text { water } \\
\text { (feet) }\end{array}$} & \multicolumn{7}{|c|}{ Samples collected for analyses } \\
\hline & & & & & & & AVC & NUT & TM & PST & voc & svoc & ISO \\
\hline 1 & 182630662740 & Manatí & Atenas & PS & 240 & 150 & $\mathrm{Y}$ & $\mathrm{Y}$ & $\mathrm{Y}$ & $\mathrm{Y}$ & $\mathrm{Y}$ & $\mathrm{Y}$ & $\mathrm{Y}$ \\
\hline 2 & 182613663414 & Vega Baja & Alturas & PS & -- & 220 & $\mathbf{Y}$ & $\mathrm{Y}$ & $\mathrm{Y}$ & $\mathrm{Y}$ & $\mathbf{Y}$ & $\mathrm{Y}$ & $\mathrm{Y}$ \\
\hline 3 & 182328662529 & Vega Baja & Beauchamp & AG & -- & 35 & $\mathrm{Y}$ & $\mathrm{Y}$ & $\mathrm{Y}$ & $\mathrm{Y}$ & $\mathrm{Y}$ & $\mathbf{Y}$ & $\mathrm{Y}$ \\
\hline 4 & 182751662937 & Manatí & Boquillas & PS & 132 & 41 & Y & $\mathrm{Y}$ & $\mathrm{Y}$ & $\mathrm{Y}$ & $\mathbf{Y}$ & $\mathrm{Y}$ & $\mathrm{Y}$ \\
\hline 5 & 182320662552 & Vega Baja & Cátala & LV & 150 & - & $\mathrm{Y}$ & $\mathrm{Y}$ & $\mathrm{Y}$ & $\mathrm{Y}$ & $\mathrm{Y}$ & $\mathrm{N}$ & $\mathrm{Y}$ \\
\hline 6 & 182540662754 & Manatí & Coto Sur \# I & PS & 300 & 233 & $\mathrm{Y}$ & $\mathrm{Y}$ & $\mathrm{Y}$ & $\mathrm{Y}$ & $\mathrm{N}$ & $\mathrm{N}$ & $\mathrm{Y}$ \\
\hline 7 & 182552662647 & Manatí & Coto Sur \#2 & $\mathrm{U}$ & 390 & 260 & $\mathrm{~N}$ & $\mathrm{Y}$ & $\mathbf{N}$ & $\mathrm{Y}$ & $\mathrm{Y}$ & $\mathrm{N}$ & $\mathrm{Y}$ \\
\hline 8 & 182554662749 & Manatí & Coto Sur \#3 & $\mathrm{U}$ & 215 & 240 & $\mathrm{~N}$ & $\mathrm{Y}$ & $\mathrm{N}$ & $\mathrm{Y}$ & $\mathrm{Y}$ & $\mathrm{N}$ & $\mathrm{Y}$ \\
\hline 9 & 182546662712 & Manatí & Coto Sur \#5 & PS & 500 & 267 & $\mathrm{Y}$ & $\mathrm{Y}$ & $\mathrm{Y}$ & $\mathrm{Y}$ & $\mathrm{Y}$ & $\mathrm{Y}$ & $\mathrm{Y}$ \\
\hline 10 & 182542662736 & Manatí & Coto Sur \#6 & PS & 475 & 244 & $\mathrm{Y}$ & $\mathrm{Y}$ & Y & $\mathrm{Y}$ & $\mathrm{Y}$ & $\mathrm{Y}$ & $\mathrm{Y}$ \\
\hline 11 & 182546662730 & Manatí & Coto Sur WH & AG & -- & 258 & $\mathbf{Y}$ & $\mathrm{Y}$ & $\mathrm{Y}$ & $\mathrm{Y}$ & $\mathrm{Y}$ & $\mathrm{Y}$ & $\mathrm{Y}$ \\
\hline 12 & 182617662902 & Manatí & Cordova Dávila & PS & 200 & 92 & $\mathrm{Y}$ & $\mathrm{Y}$ & $\mathrm{Y}$ & $\mathrm{Y}$ & $\mathrm{Y}$ & $\mathrm{Y}$ & $\mathrm{Y}$ \\
\hline 13 & 182705662952 & Manatí & Cruz Rosa Rivas & PS & - & 118 & $\mathrm{Y}$ & $\mathrm{Y}$ & $\mathrm{Y}$ & $\mathrm{Y}$ & $\mathrm{Y}$ & $\mathrm{Y}$ & $\mathrm{Y}$ \\
\hline 14 & 182701662902 & Manatí & Jacinto Cubano & LV & -- & -- & $\mathbf{Y}$ & $Y$ & $\mathrm{Y}$ & $\mathrm{Y}$ & $\mathrm{Y}$ & $\mathrm{Y}$ & $\mathrm{Y}$ \\
\hline 15 & 182530662854 & Manatí & Escalfullery & PS & 400 & 225 & $\mathrm{~N}$ & $\mathrm{Y}$ & $\mathrm{N}$ & $\mathrm{N}$ & $\mathrm{Y}$ & $\mathrm{N}$ & $\mathrm{N}$ \\
\hline 16 & 182646662359 & Vega Baja & Vega Baja \#4 & PS & -- & 76 & $\mathrm{Y}$ & $\mathrm{Y}$ & Y & $\mathrm{Y}$ & $\mathrm{Y}$ & $\mathrm{N}$ & $\mathrm{Y}$ \\
\hline 17 & 182638662722 & Manatí & Marista & $\mathrm{U}$ & - & - & $\mathrm{N}$ & $\mathrm{Y}$ & $\mathrm{N}$ & $\mathrm{Y}$ & $\mathrm{Y}$ & $\mathrm{Y}$ & $\mathrm{Y}$ \\
\hline 18 & 182412662949 & Manatí & Monserrate Sur & LV & 180 & 29 & $\mathrm{Y}$ & $\mathrm{N}$ & Y & $\mathrm{Y}$ & $\mathrm{N}$ & $\mathrm{Y}$ & $\mathrm{Y}$ \\
\hline 19 & 182514662901 & Manatí & Mónaco & PS & 500 & 250 & $\mathbf{N}$ & $\mathrm{Y}$ & $\mathrm{N}$ & $\mathrm{Y}$ & $\mathbf{Y}$ & $\mathrm{N}$ & $\mathrm{Y}$ \\
\hline 20 & 182735662343 & Vega Baja & NC-9 & $\mathrm{UA}$ & 1,540 & 10 & $\mathbf{Y}$ & $\mathrm{Y}$ & $\mathrm{Y}$ & $\mathrm{N}$ & $\mathrm{Y}$ & $\mathrm{Y}$ & $\mathbf{Y}$ \\
\hline 21 & 182657662506 & Vega Baja & Ojo de Agua & SP & - & - & $\mathrm{N}$ & $\mathrm{N}$ & $\mathrm{N}$ & $\mathrm{Y}$ & $\mathrm{N}$ & $\mathrm{Y}$ & $\mathrm{Y}$ \\
\hline 22 & 182604662925 & Manatí & Manatí 3 & PS & 200 & 63 & $\mathbf{Y}$ & $\mathrm{Y}$ & $\mathbf{Y}$ & $\mathrm{Y}$ & $\mathrm{Y}$ & $\mathrm{N}$ & $\mathbf{Y}$ \\
\hline 23 & 182553662429 & Vega Baja & Pugnado \#1 & PS & 150 & - & $\mathrm{Y}$ & $\mathrm{Y}$ & $\mathrm{Y}$ & $\mathrm{Y}$ & $\mathrm{Y}$ & $\mathrm{Y}$ & $\mathrm{Y}$ \\
\hline 24 & 182545662438 & Vega Baja & Pugnado \#2 & PS & 340 & 242 & $\mathrm{Y}$ & $\mathrm{Y}$ & $\mathrm{Y}$ & $\mathrm{Y}$ & $\mathrm{Y}$ & $\mathrm{Y}$ & $\mathbf{Y}$ \\
\hline 25 & 182615662735 & Manatí & Procter \& Gamble & IN & 240 & 174 & $\mathbf{Y}$ & $\mathrm{Y}$ & Y & $\mathrm{Y}$ & $\mathrm{Y}$ & $\mathrm{Y}$ & $\mathbf{Y}$ \\
\hline 26 & 182305662659 & Manatí & Quebrada & SW & -- & - & $\mathrm{N}$ & $\mathrm{Y}$ & $\mathbf{N}$ & $\mathrm{Y}$ & $\mathrm{Y}$ & $\mathrm{Y}$ & $\mathrm{Y}$ \\
\hline 27 & 182714662924 & Manatí & Rabanos & PS & 160 & 112 & $\mathrm{Y}$ & $\mathrm{Y}$ & $\mathbf{Y}$ & $\mathrm{N}$ & $\mathbf{Y}$ & $\mathrm{Y}$ & $\mathrm{Y}$ \\
\hline 28 & 182316662737 & Manạii & Río Arriba \#2 & PS & 300 & 38 & $\mathrm{Y}$ & $\mathrm{Y}$ & $\mathbf{Y}$ & $\mathrm{Y}$ & $\mathrm{Y}$ & $\mathrm{N}$ & $\mathrm{Y}$ \\
\hline 29 & 182312662749 & Manatí & Río Arriba \#3 & PS & 220 & 55 & Y & $\mathrm{Y}$ & $\mathrm{Y}$ & $\mathbf{Y}$ & $\mathbf{Y}$ & $\mathrm{N}$ & Y \\
\hline 30 & 182651662416 & Vega Baja & Vega Baja \#2 & PS & 120 & 76 & $\mathbf{Y}$ & $\mathrm{Y}$ & $\mathbf{Y}$ & $\mathrm{Y}$ & $\mathbf{Y}$ & $\mathbf{Y}$ & Y \\
\hline 31 & 182644662405 & Vega Baja & Vega Baja \#3 & PS & 140 & -- & $\mathrm{Y}$ & $\mathbf{Y}$ & $\mathrm{Y}$ & $\mathbf{Y}$ & $\mathrm{Y}$ & $\mathrm{Y}$ & $\mathrm{Y}$ \\
\hline
\end{tabular}




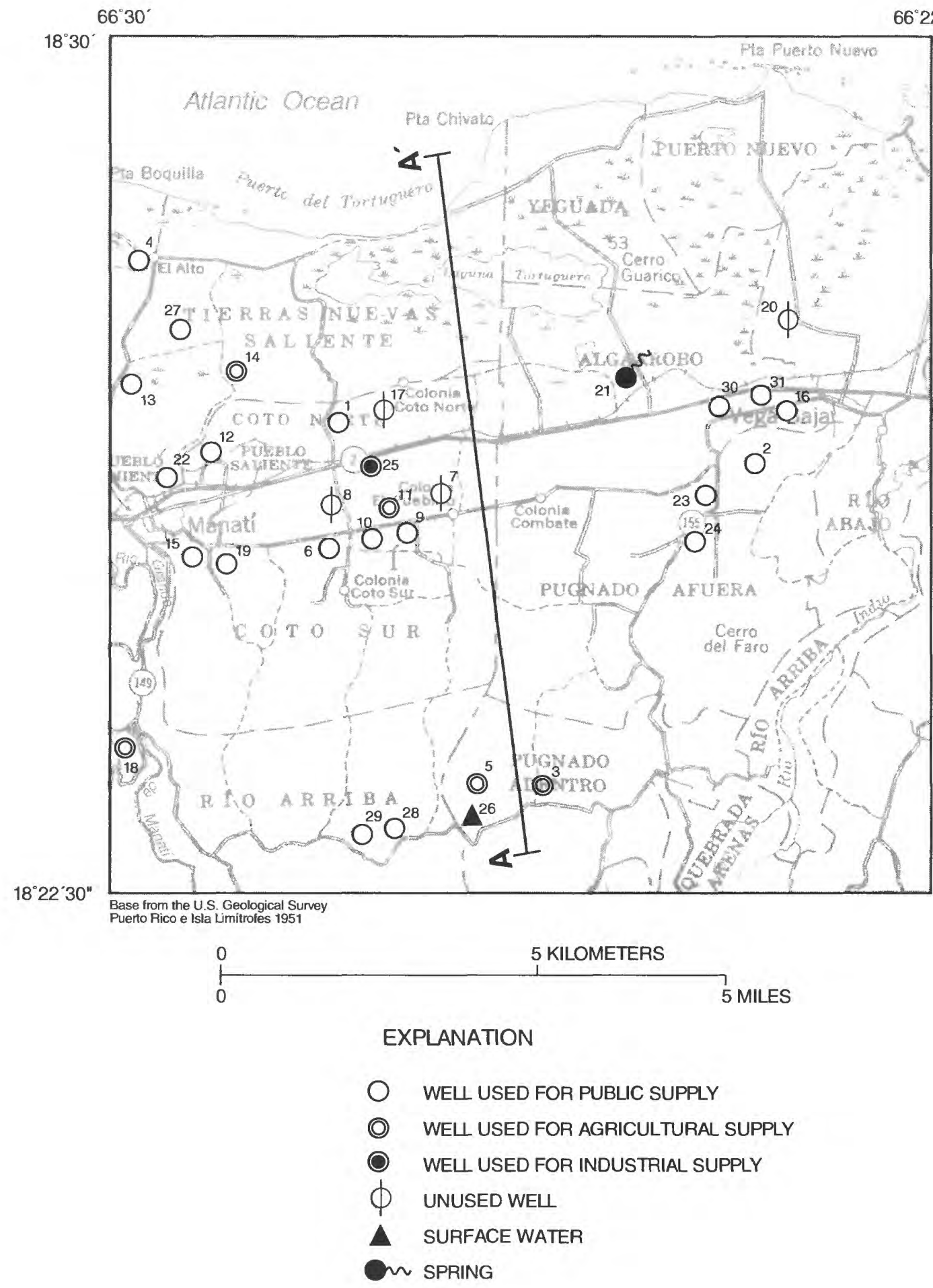

Figure 3. Location of sampling sites in the Manatí quadrangle, Puerto Rico. 
temperature, specific conductance, and $\mathrm{pH}$ were determined during pumping at the nearest discharge tap from the well head. Samples for the analyses of alkalinity, the common constituents, nutrients, trace metals, and stable isotopes were withdrawn from a churn splitter (a container commonly used to obtain representative sub-samples) filled at the point of sampling. Samples for the analyses of insecticides, organic compounds (volatile and semivolatile) and total organic carbon were collected directly into sample containers at the well-discharge points. Sample treatment and preservation was conducted on-site following standard USGS procedures.

The samples were shipped to and analyzed at the U.S. Geological Survey Central Laboratory in Arvada, Colorado. Analytical results indicated as "dissolved" or "filtered sample" correspond to samples filtered through a 0.45 micron filter.

\section{RESULTS}

Analytical results of the field and laboratory measurements of selected physical properties, major ions, nutrients, and total organic carbon are summarized in table 2. Analytical results for laboratory measurements of trace metals are summarized in table 3. Pesticides and organic compounds analyzed at selected wells are summarized in table 4, and analytical results are presented in table 5. Stable isotope analytical results are summarized in table 6. 
Table 2. Major ions, nutrients, total organic carbon, and physical properties at selected surface- and ground-water sampling sites in the Manatí quadrangle, Puerto Rico

[SC, specific conductance; $\mu \mathrm{S} / \mathrm{cm}$, microsiemens per centimeter; $\mathrm{pH}$, whole water field determinations; Temp, water temperature; ${ }^{\circ} \mathrm{C}$, degrees Celsius; mg/L; milligrams per liter; $\mathrm{Ca}$, calcium; $\mathrm{Mg}$, magnesium; $\mathrm{Na}$, sodium; $\mathrm{K}$, potassium; $\mathrm{HCO}_{3}$, bicarbonate, field end-point titration whole water sample; --, no data available; (a), artesian; (b), spring; (c) surface-water sample; all analytical results are for filtered water samples except alkalinity and TOC]

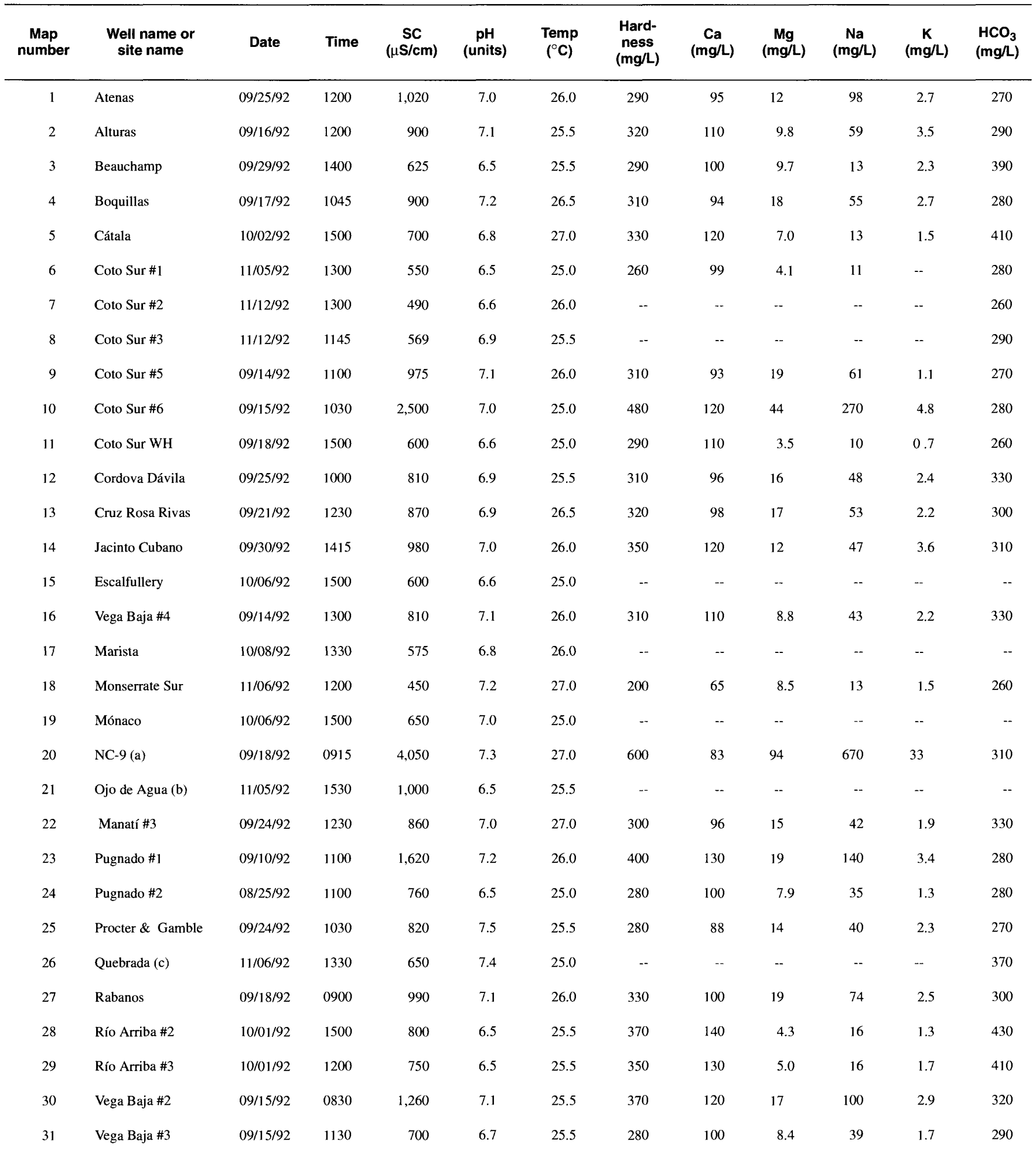


Table 2. Major ions, nutrients, total organic carbon, and physical properties at selected surface and ground-water sampling sites in the Manatí quadrangle, Puerto Rico--Continued

[Alk, alkalinity; $\mathrm{mg} / \mathrm{L}$; milligrams per liter; $\mathrm{CaCO}_{3}$; calcium carbonate; $\mathrm{Cl}$, chloride; $\mathrm{F}$, fluoride; $\mathrm{Br}$, bromide; $\mathrm{SiO}_{2}$, silica; $\mathrm{DS}$, dissolved solids sum of constituents; $\mathrm{NO}_{2}$, nitrite as $\mathrm{N} ; \mathrm{NO}_{2}+\mathrm{NO}_{3}$, nitrite plus nitrate as $\mathrm{N} ; \mathrm{NH}_{4}$, ammonia as $\mathrm{N} ; \mathrm{P}$, phosphorus; o-P, orthophosphate; TOC, total organic carbon; --, no data available; <, less than; (a), artesian; (b), spring; (c) surface water sample; all analytical results are for filtered water samples except alkalinity and TOC]

\begin{tabular}{|c|c|c|c|c|c|c|c|c|c|c|c|c|c|c|}
\hline $\begin{array}{c}\text { Map } \\
\text { number }\end{array}$ & $\begin{array}{l}\text { Well name or } \\
\text { site name }\end{array}$ & $\begin{array}{c}\text { Alk } \\
(\mathrm{mg} / \mathrm{L} \\
(\mathrm{as} \\
\left.\left.\mathrm{CaCO_{3 }}\right)\right)\end{array}$ & $\begin{array}{c}\mathrm{SO}_{4} \\
(\mathrm{mg} / \mathrm{L})\end{array}$ & $\underset{(\mathrm{mg} / \mathrm{L})}{\mathrm{Cl}}$ & $\underset{(m g / L)}{F}$ & $\begin{array}{c}\mathrm{Br} \\
(\mathrm{mg} / \mathrm{L})\end{array}$ & $\begin{array}{c}\mathrm{SiO}_{2} \\
\text { (mg/L) }\end{array}$ & $\begin{array}{c}\text { DS } \\
(\mathrm{mg} / \mathrm{L})\end{array}$ & $\begin{array}{c}\mathrm{NO}_{2} \\
(\mathrm{mg} / \mathrm{L})\end{array}$ & $\begin{array}{c}\mathrm{NO}_{2}+\mathrm{NO}_{3} \\
(\mathrm{mg} / \mathrm{L})\end{array}$ & $\begin{array}{c}\mathrm{NH}_{4} \\
(\mathrm{mg} / \mathrm{L})\end{array}$ & $\underset{(\mathrm{mg} / \mathrm{L})}{\mathbf{P}}$ & $\begin{array}{c}\text { o-P } \\
(\mathrm{mg} / \mathrm{L})\end{array}$ & $\begin{array}{c}\text { TOC } \\
\text { (mg/L) }\end{array}$ \\
\hline 1 & Atenas & 220 & 29 & 180 & $<.10$ & 0.63 & 7.8 & 563 & $<.010$ & 6.5 & 0.020 & 0.020 & 0.020 & 0.7 \\
\hline 2 & Alturas & 240 & 26 & 120 & $<.10$ & .40 & 7.0 & 481 & $<.010$ & 3.8 & .020 & .010 & .010 & .5 \\
\hline 3 & Beauchamp & 320 & 8.4 & 21 & .20 & .08 & 14 & 360 & $<.010$ & .40 & .020 & .020 & $<.010$ & .3 \\
\hline 4 & Boquillas & 230 & 22 & 120 & $<.10$ & .44 & 19 & 472 & $<.010$ & 3.8 & .020 & .030 & .020 & .7 \\
\hline 5 & Cátala & 340 & 23 & 25 & .10 & .11 & 11 & 403 & $<.010$ & 1.2 & .010 & .020 & $<.010$ & .8 \\
\hline 6 & Coto Sur \#1 & 230 & 3 & 17 & $<.10$ & -- & 6.9 & -- & $<.010$ & 12 & .020 & .010 & $<.010$ & .5 \\
\hline 7 & Coto Sur \#2 & 210 & -- & -- & -- & -- & -- & -- & $<.010$ & 14 & .010 & $<.010$ & $<.010$ & -- \\
\hline 8 & Coto Sur \#3 & 240 & -- & -- & -- & -- & -- & -- & $<.010$ & 7.5 & .020 & $<.010$ & $<.010$ & -- \\
\hline 9 & Coto Sur \#5 & 220 & 15 & 160 & $<.10$ & .36 & 6.7 & 488 & -- & -- & -- & -- & -- & .2 \\
\hline 10 & Coto Sur \#6 & 230 & -- & -- & -- & -- & -- & -- & -- & -- & -- & -- & -- & .3 \\
\hline 11 & Coto Sur WH & 210 & 8.6 & 16 & $<10$ & .07 & 6.7 & 301 & $<.010$ & 18 & .010 & .010 & .010 & .6 \\
\hline 12 & Cordova Dávila & 270 & 15 & 83 & $<.10$ & .32 & 8.3 & 437 & $<.010$ & 6.1 & .030 & .020 & .020 & .5 \\
\hline 13 & Cruz Rosa Rivas & 250 & 24 & 120 & $<.10$ & .46 & 20 & 484 & $<.010$ & 2.7 & .070 & .040 & .030 & .3 \\
\hline 14 & Jacinto Cubano & 260 & 22 & 110 & $<.10$ & .37 & 11 & 488 & $<.010$ & 11 & .030 & .020 & .010 & .4 \\
\hline 15 & Escalfullery & -- & -- & -- & -- & -- & -- & -- & $<.010$ & 5.7 & .400 & .020 & .010 & -- \\
\hline 16 & Vega Baja \#4 & 270 & 22 & 94 & $<.10$ & .35 & 8.4 & 450 & -- & -- & -- & -- & -- & .4 \\
\hline 17 & Marista & -- & & -- & -- & -- & -- & -- & $<.010$ & 6.9 & .020 & .020 & .010 & .3 \\
\hline 18 & Monserrate Sur & 210 & 11 & 13 & .20 & .07 & 18 & 258 & -- & -- & -- & -- & -- & -- \\
\hline 19 & Mónaco & -- & -- & -- & -- & -- & -- & -- & $<.010$ & 6.2 & $<.010$ & $<.010$ & $<.010$ & .9 \\
\hline 20 & NC-9 (a) & 260 & 180 & 1,200 & .40 & -- & 13 & 2,420 & $<.010$ & 0.08 & .10 & $<.010$ & $<.010$ & .5 \\
\hline 21 & Ojo de Agua (b) & -- & -- & -- & -- & -- & -- & -- & -- & -- & -- & -- & -- & -- \\
\hline 22 & Manatí \#3 & 270 & 22 & 84 & $<.10$ & .30 & 17 & 445 & $<.010$ & 5.2 & .050 & .020 & .020 & .4 \\
\hline 23 & Pugnado \# 1 & 230 & 43 & 330 & $<.10$ & .14 & 6.9 & 814 & $<.010$ & 4.0 & .030 & $<.010$ & $<.010$ & .4 \\
\hline 24 & Pugnado \#2 & 230 & 10 & 92 & $<.10$ & .29 & 6.7 & 398 & $<.010$ & 7.0 & .030 & $<.010$ & $<.010$ & 2.6 \\
\hline 25 & Procter \& Gamble & 220 & 11 & 80 & .10 & .28 & 6.5 & 383 & $<.010$ & 9.2 & .050 & .010 & $<.010$ & .3 \\
\hline 26 & Quebrada (c) & 300 & -- & -- & -- & -- & -- & -- & .010 & .4 & .160 & .030 & .020 & 8.7 \\
\hline 27 & Rabanos & 250 & 27 & 160 & $<.10$ & .59 & 17 & 551 & $<.010$ & 3.6 & $<.010$ & .030 & .030 & .5 \\
\hline 28 & Río Arriba \#2 & 350 & 37 & 36 & .10 & .15 & 11 & 458 & $<.010$ & 1.5 & .010 & $<.010$ & $<.010$ & .7 \\
\hline 29 & Río Arriba \#3 & 340 & 32 & 33 & .10 & .14 & 10 & 431 & $<.010$ & 2.3 & $<.010$ & $<.010$ & $<.010$ & 1.2 \\
\hline 30 & Vega Baja \#2 & 260 & 35 & 240 & $<.10$ & .74 & 8.2 & 681 & -- & -- & -- & -- & -- & .4 \\
\hline 31 & Vega Baja \#3 & 240 & 20 & 82 & $<.10$ & .30 & 6.5 & 400 & -- & -- & -- & -- & -- & .5 \\
\hline
\end{tabular}




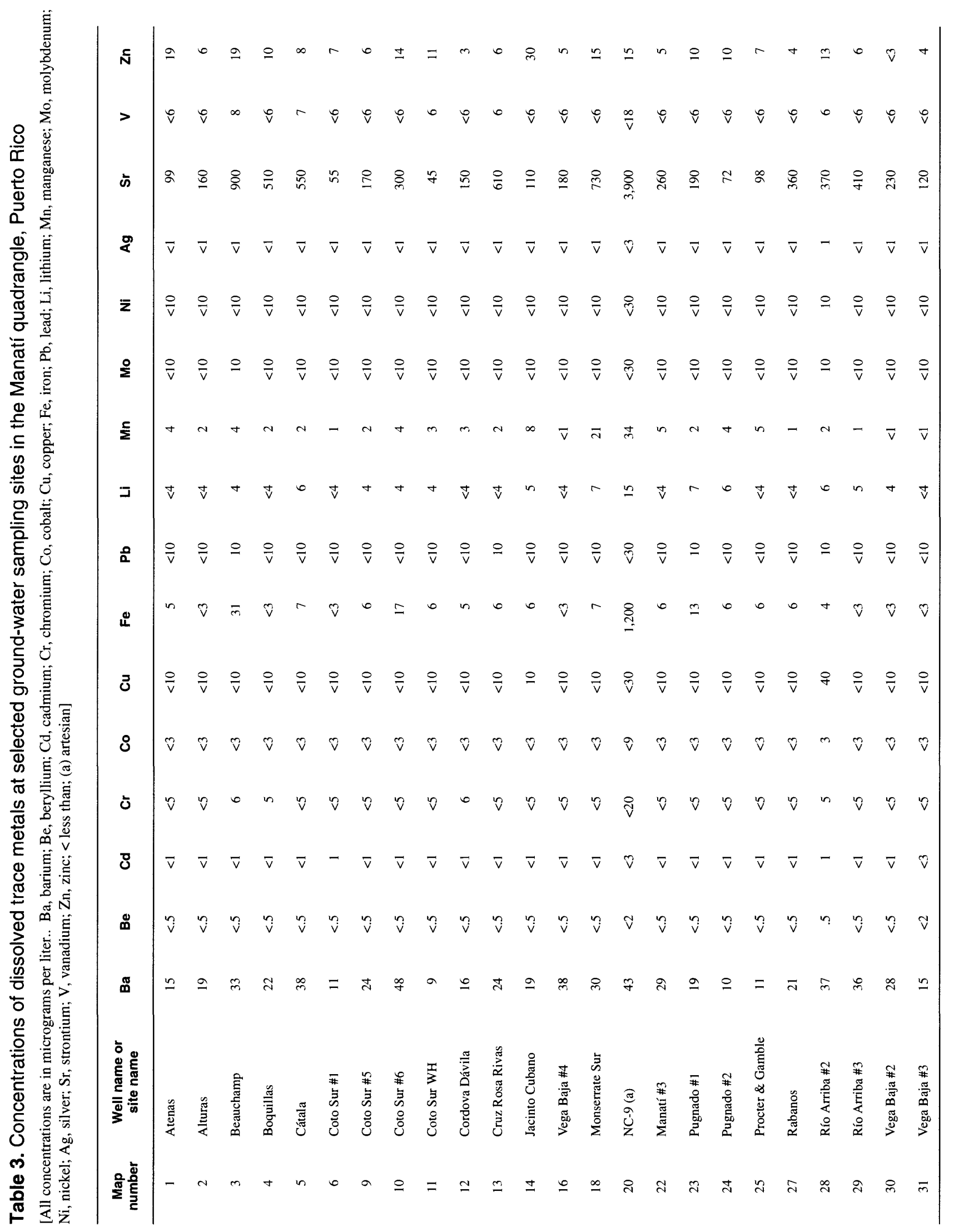


Table 4. Organic compounds analyzed in water samples collected at selected sampling sites in the Manatí quadrangle, Puerto Rico

[All concentrations are in micrograms per liter, except where indicated and as total constituents. MRL, minimum reporting level.]

\begin{tabular}{lclr}
\hline \multicolumn{2}{c}{ I. Organochlorine pesticides and related compounds } & \\
Compound & MRL & Compound & MRL \\
\hline Aldrin & 0.01 & Fonofos & 0.01 \\
Chlordane & .01 & Gross PCB's & .01 \\
Chlorpyrifor & .01 & Gross PCN's & .10 \\
DDD & .01 & Heptachlor & .01 \\
DDE & .01 & Heptachlor Epoxide & .01 \\
DDT & .01 & Lindane & .01 \\
DEF & .01 & Methoxychlor & .01 \\
Di-Systone & .01 & Mirex & .01 \\
Dieldrin & .01 & Perthane & .10 \\
Endosulfan & .01 & Phorate & .01 \\
Endrin & .01 & Toxaphene & 1.00 \\
\hline
\end{tabular}

II. Organophosphorus pesticides

\begin{tabular}{lclc} 
Compound & MRL & Compound & MRL \\
\hline Diazinon & .01 & Methyl Parathion & .01 \\
Ethion & .01 & Parathion & .01 \\
Melathion & .01 & Trithion & .01 \\
\hline
\end{tabular}

III. Volatile organic chemicals (VOCs)

\begin{tabular}{lclc} 
Compound & MRL & Compound & MRL \\
\hline 1,1,1,2-Tetrachloroethane & 3 & 1,2-Dichlorobenzene & 3 \\
1,1,1,-Trichloroethane & 3 & 1,2-Dichloroethane & 3 \\
1,1,2,2-Tetrachloroethane & 3 & 1,2-Dichloropropane & 3 \\
1,1,2-Trichloroethane & 3 & 1,2-Transdichloroethene & 3 \\
1,1-Dichloroethane & 3 & 1,3,5-Trimethylbenzene & 3 \\
1,1-Dichloroethylene & 3 & 1,3-Dichlorobenzene & 3 \\
1,1-Dichloropropene & 3 & 1,3-Dichloropropane & 3 \\
1,2,3-Trichlorobenzene & 3 & 1,4-Chlorotoluene & 3 \\
1,2,3-Trichloropropane & 3 & 1,4-Dichlorobenzene & 3 \\
1,2,4-Trichlorobenzene & 3 & 2,2-Dichloropropane & 3 \\
1,2,4-Trimethylbenzene & 3 & 2-Chloroethylvinylether & 3
\end{tabular}


Table 4. Organic compounds analyzed in water samples collected at selected sampling sites in the Manatí quadrangle, Puerto Rico--Continued

\begin{tabular}{|c|c|c|c|}
\hline \multicolumn{4}{|c|}{ III. Volatile organic chemicals (VOCs)--Continued } \\
\hline Compound & MRL & Compound & MRL \\
\hline 1,2-Chlorotoluene & 3 & Acrolein & 20 \\
\hline 1,2-Dibromoethane & 3 & Acrylonitrile & 20 \\
\hline Benzene & 3 & Methylchloride & 3 \\
\hline Bromobenzene & 3 & Methylene chloride & 3 \\
\hline Bromochloromethane & 3 & Methyltertbutylether & 3 \\
\hline Bromoform & 3 & N-Butylbenzene & 3 \\
\hline Carbontetrachloride & 3 & N-propylbenzene & 3 \\
\hline Chlorobenzene & 3 & Naphthalene & 3 \\
\hline Chlorodibromomethane & 3 & P-isopropyltoluene & 3 \\
\hline Chloroethane & 3 & Sec-butylbenzene & 3 \\
\hline Chloroform & 3 & Styrene & 3 \\
\hline Cis-1,2-dichloroethene & 3 & Tert-butylbenzene & 3 \\
\hline Cis-1,3-dichloropropene & 3 & Tetrachloroethylene & 3 \\
\hline Dibromochloropropane & 3 & Toluene & 3 \\
\hline Dibromomethane & 3 & Trans-1,3-dichloropropene & 3 \\
\hline Dichlorobromomethane & 3 & Trichloroethylene & 3 \\
\hline Dichlorodifluoromethane & 3 & Trichlorofluoromethane & 3 \\
\hline Ethylbenzene & 3 & Trichlorotrifluoroethane & 3 \\
\hline Hexachlorobutadiene & 3 & Vinyl chloride & 1 \\
\hline Isopropylbenzene & 3 & Xylenes,total ortho, meta, & \\
\hline Methylbromide & 3 & and para & 3 \\
\hline \multicolumn{4}{|c|}{ IV. Semivolatile organic chemicals } \\
\hline Compound & MRL & Compound & MRL \\
\hline 1,2,4-Trichlorobenzene & 5 & 2-Chlorophenol & 5 \\
\hline 1,2,5,6-Dibenzanthracene & 10 & 2-Nitrophenol & 5 \\
\hline 1,2-Dichlorobenzene & 5 & 3,3-Dichlorobenzidine & 20 \\
\hline 1,2-Diphenylhydrazine & 5 & 2-Methyl-4,6-dinitrophenol & 30 \\
\hline 1,3-Dichlorobenzene & 5 & 4-Bromophenylphenylether & 5 \\
\hline 1,4-Dichlorobenzene & 5 & 4-Chlorophenylphenylether & 5 \\
\hline
\end{tabular}


Table 4. Organic compounds analyzed in water samples collected at selected sampling sites in the Manatí quadrangle, Puerto Rico--Continued

\section{Semivolatile organic chemicals--Continued}

\begin{tabular}{lrlr} 
Compound & MRL & Compound & MRL \\
\hline 2,4,6-Trichlorophenol & 20 & 4-Nitrophenol & 30 \\
2,4-Dichlorophenol & 5 & Acenaphthene & 5 \\
2,4-Dimethylphenol & 5 & Acenaphthylene & 5 \\
2,4-Dinitrophenol & 20 & Anthracene & 5 \\
2,4-Dinitrotoluene & 5 & Benzidine & 40 \\
2,6-Dinitrotoluene & 5 & Benzo (a) pyrene & 10 \\
2-Chloronaphthalene & 5 & Benzo (b) fluoranthene & 10 \\
Benzo (k) fluoranthene & 10 & Fluorene & 5 \\
Benzo (a) anthracene & 10 & Hexachlorobenzene & 5 \\
Benzo (g,h,i) perylene & 10 & Hexachlorobutadeine & 5 \\
Bis (2-chloroethoxy) methane & 5 & Hexachlorocyclopentadeine & 5 \\
Bis (2-chloroethyl) ether & 5 & Hexachloroethane & 5 \\
Bis (2-chloroisopropyl) ether & 5 & Indeno (1,2,3-cd) pyene & 10 \\
Bis (2-ethylhexyl) phthalate & 5 & Isophorone & 5 \\
Butyl benzyl phthalate & 5 & N-Nitrosodi-n-propylamine & 5 \\
4-Chloro-3-methylphenol & 30 & N-Nitrosodiphenylamine & 5 \\
Chrysene & 10 & Naphthalene & 5 \\
Di-n-butyl phthalate & 5 & Nitrobenzene & 5 \\
Di-n-octyl phthalate & 10 & Pentachlorophenol & 5 \\
Diethyl phthalate & 5 & Phenantherene & 5 \\
Dimethyl phthalate & 5 & Phenol & 5 \\
Fluoranthene & 5 & Pyrene & 5 \\
\hline & & & 5 \\
\hline
\end{tabular}


Table 5. Pesticides and organic compounds detected in water from selected wells in the Manatí quadrangle, Puerto Rico

[All concentration are in micrograms per liter]

\begin{tabular}{|c|c|c|}
\hline Well number & Compound & Concentration \\
\hline \multirow[t]{2}{*}{1} & Dieldrin & 0.20 \\
\hline & DDD & 0.01 \\
\hline \multirow[t]{2}{*}{2} & Dieldrin & 0.01 \\
\hline & Heptachlor epoxide & 0.01 \\
\hline \multirow[t]{2}{*}{4} & Dieldrin & 0.01 \\
\hline & Heptachlor epoxide & 0.01 \\
\hline \multirow[t]{3}{*}{6} & Lindane & 0.01 \\
\hline & Toxaphene & 1.00 \\
\hline & Heptachlor epoxide & 0.02 \\
\hline 7 & Dieldrin & 0.12 \\
\hline \multirow[t]{2}{*}{8} & Dieldrin & 0.07 \\
\hline & 1,2,3,-Trichloropropane & 3.20 \\
\hline 9 & Dieldrin & 0.06 \\
\hline 10 & Dieldrin & 0.04 \\
\hline \multirow[t]{2}{*}{11} & Dieldrin & 0.24 \\
\hline & Toxaphene & 6.00 \\
\hline \multirow[t]{2}{*}{12} & Dieldrin & 0.01 \\
\hline & Heptachlor epoxide & 0.01 \\
\hline \multirow[t]{2}{*}{13} & Dieldrin & 0.01 \\
\hline & Heptachlor epoxide & 0.01 \\
\hline 17 & Dieldrin & 0.01 \\
\hline 18 & Diazinon & 0.01 \\
\hline 19 & Dieldrin & 0.01 \\
\hline 23 & Dieldrin & 0.06 \\
\hline 24 & Diazinon & 0.01 \\
\hline \multirow[t]{2}{*}{25} & Dieldrin & 0.12 \\
\hline & Toxaphene & 1.00 \\
\hline
\end{tabular}


Table 6. Stable isotope analyses at selected ground-water sampling sites in the Manatí quadrangle, Puerto Rico

[O-18, oxygen-18; H-2 deuterium; N-15, nitrogen-15; S-34, sulfur-34; O-18 and H-2 in parts per mil relative to Vienna Standard Mean Ocean Water (VSMOW); N-15 in parts per mil relative to air; S-34 in sulfate, aqueous in parts per mil relative to Canyon Diablo Troilite (CDT); (a), artesian; (b), spring; (c), surface-water sample]

\begin{tabular}{|c|c|c|c|c|c|}
\hline Map number & $\begin{array}{l}\text { Well name or } \\
\text { site name }\end{array}$ & $0-18$ & $\mathrm{H}-2$ & $\mathrm{~N}-15$ & S-34 \\
\hline 1 & Atenas & -2.60 & -8.5 & 4.5 & 20.0 \\
\hline 2 & Alturas & -2.60 & -9.5 & 8.2 & 13.1 \\
\hline 3 & Beauchamp & -2.50 & -7.0 & 7.0 & -- \\
\hline 4 & Boquillas & -2.60 & -8.5 & 11.6 & 13.7 \\
\hline 5 & Cátala & -2.45 & -7.0 & 10.0 & 2.4 \\
\hline 6 & Coto Sur \#1 & -2.55 & -8.0 & 3.8 & -- \\
\hline 7 & Coto Sur \#2 & -2.58 & -7.7 & 3.4 & -- \\
\hline 8 & Coto Sur \#3 & -2.88 & -11.1 & 3.8 & 16.3 \\
\hline 9 & Coto Sur \#5 & -2.65 & -9.0 & 3.4 & 15.4 \\
\hline 10 & Coto Sur \#6 & -2.50 & -7.0 & 3.1 & 19.2 \\
\hline 11 & Coto Sur WH & -2.45 & -8.0 & 2.5 & 11.5 \\
\hline 12 & Cordova Dávila & -2.45 & -7.5 & 8.1 & 17.4 \\
\hline 13 & Cruz Rosa Rivas & -2.65 & -8.5 & 11.9 & 13.5 \\
\hline 14 & Jacinto Cubano & -2.55 & -9.5 & 10.9 & 16.6 \\
\hline 16 & Vega Baja \#4 & -2.50 & -9.0 & 8.9 & 11.3 \\
\hline 17 & Marista & -- & -- & 5.4 & 19.1 \\
\hline 18 & Monserrate Sur & -2.70 & -9.5 & 9.5 & 3.9 \\
\hline 19 & Mónaco & -2.55 & -8.5 & 6.0 & 7 \\
\hline 20 & NC-9 (a) & -1.50 & -1.0 & -- & 19.6 \\
\hline 21 & Ojo de Agua (b) & -2.40 & -7.5 & 8.8 & 13.4 \\
\hline 22 & Manatí \#3 & -2.60 & -8.0 & 12.7 & 16.1 \\
\hline 23 & Pugnado \#1 & -2.55 & -9.5 & -- & 17.6 \\
\hline 24 & Pugnado \#2 & -- & -- & -- & 14.1 \\
\hline 25 & Procter \& Gamble & -2.50 & -7.5 & 3.8 & 18.5 \\
\hline 26 & Quebrada (c) & -2.40 & -6.5 & 7.2 & 21.9 \\
\hline 27 & Rabanos & -2.65 & -8.0 & 12.0 & 17.2 \\
\hline 28 & Río Arriba \#2 & -2.55 & -10.0 & 14.3 & 2.5 \\
\hline 29 & Río Arriba \#3 & -2.50 & -7.5 & 13.4 & -- \\
\hline 30 & Vega Baja \#2 & -2.50 & -8.5 & 7.2 & 16.5 \\
\hline 31 & Vega Baja \#3 & -2.55 & -8.0 & 7.6 & 11.9 \\
\hline
\end{tabular}




\section{REFERENCES}

Bennett, G.D., and Giusti, E.V., 1972, Ground water in the Tortuguero area, Puerto Rico: U.S. Geological Survey Bulletin 10, 25 p.

Brown, Eugene, Skougstad, M.W., and Fishman, M.J.,1970, Methods for the collection and analysis of water samples for dissolved minerals and gases: Techniques of WaterResources Investigations of the U.S. Geological Survey, Book 5, Chapter A1, $160 \mathrm{p}$.

Goerlitz, D.F., and Brown; E., 1972, Methods for analysis of organic substances in water: Techniques of Water-Resources Investigations of the U.S. Geological Survey, Book 5, Chapter A3, 40 p.

Hem, J.D., 1989, Study and interpretation of the chemical characteristics of natural water $(3 \mathrm{~d}$ ed.): U. S. Geological Survey Water-Supply Paper 2254, 263 p.

Monroe, W.H., 1980, Geology of the Middle Tertiary Formations of Puerto Rico: U.S. Geological Survey Professional Paper 953, $90 \mathrm{p}$.

Rodríguez-Martínez, Jesús, 1995, Hydrogeology of the North Coast Limestone aquifer system of Puerto Rico: U.S. Geological Survey Water-Resources Investigations Report 94-4249, 22 p.
Skougstad, M.W., Fishman, M.J., Friedman, L.C., Erdmann, D.E., and Duncan, S.S., 1979, Methods for determination of inorganic substances in water and fluvial sediments: Techniques of Water-Resources Investigations of the U.S. Geological Survey, Book 5, Chapter A1, 626 p.

U.S. Environmental Protection Agency, 1996, Drinking water regulations and health advisories: U.S. Environmental Protection Agency, Office of Water, EPA 822-R-96-001, $11 \mathrm{p}$.

Wershaw, R.L., Fishman, M.J., and Grabbe, R.R., 1987, Methods for the determination of organic substances in water and fluvial sediments: Techniques of Water-Resources Investigations of the U.S. Geological Survey, Book 5, Chapter A3, 90 p.

Wood, W.W., 1976, Guidelines for collection and field analyses of ground-water samples for selected unstable constituents: Techniques of Water-Resources Investigations of the U.S. 。 Geological Survey, Book 1, Chapter D2, 24 p. 